Recepción: 26 / 07 / 2018

Aceptación: 16 / 08 / 2018

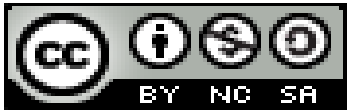

Ciencias económicas y empresariales

Publicación: 15 / 09 / 2018

Artículo de investigación

\title{
Caracterización de los modelos publicitarios del sector comercial y el cumplimiento de los derechos del consumidor
}

Characterization of the advertising models of the commercial sector and the compliance of the rights of the consumer

\section{Caracterização dos modelos de publicidade do setor comercial e conformidade dos direitos do consumidor}

\author{
Karen J. Yansapanta-Terán ${ }^{\mathrm{I}}$ \\ kyansapanta0710@uta.edu.ec \\ Leonardo G. Ballesteros-López II \\ leonardogballesteros@uta.edu.ec \\ César A. Guerrero-Velástegui III \\ ca.guerrero@uta.edu.ec3 \\ Edwin J. Santamaría-Freire IV \\ edwinjsantamaria@uta.edu
}

\section{Correspondencia: kyansapanta0710@uta.edu.ec}

${ }^{\mathrm{I}}$ Docente de la Universidad Técnica de Ambato, Ambato, Ecuador.

${ }^{\text {II }}$ Magíster en Gestión Estratégica Empresarial MBA, Ingeniero Comercial, Licenciado en ciencias Administrativas Administrador en Mercadotecnia, Docente de la Universidad Técnica de Ambato, Ambato, Ecuador.

III Magíster en Administración de Empresas Mención Planeación, Ingeniero de Empresas, Abogado de los Tribunales y Juzgados de la República del Ecuador, Docente de la Universidad Técnica de Ambato, Ambato, Ecuador.

${ }^{\mathrm{IV}}$ Magíster en Administración, Máster Universitario en Estadística Aplicada, Ingeniero en Administración de Empresas y Negocios, Docente de la Universidad Técnica de Ambato, Ambato, Ecuador. 


\title{
Resumen
}

Mediante una investigación cuantitativa, descriptiva y no exploratoria se determina cual es el modelo publicitario que tiene más relevancia en el sector comercial en el cantón de Ambato, tomando como referencia la base de datos del CIIU (Clasificación Industrial Internacional Uniforme) y el cumplimiento de los derechos del consumidor en la aplicación de los modelos que la empresa utiliza en la mima, los diez sectores comerciales más importantes del cantón son el objeto de estudio, fueron seleccionados por una muestra intencional para la proporción de información más verídica y principal, la investigación se encuentra sustentado mediante un estudio de campo.

Palabras clave: modelos publicitarios; derechos del consumidor; motivación; actitudes; calidad del producto; publicidad.

\begin{abstract}
Through quantitative, descriptive and non-exploratory research, the advertising model that is most relevant in the commercial sector in the canton of Ambato is determined, taking as reference the database of the ISIC (International Standard Industrial Classification) and compliance with the consumer rights in the application of the models that the company uses in the mima, the ten most important commercial sectors of the canton are the object of study, were selected by an intentional sample for the proportion of more truthful and main information, the research is Find supported by a field study.
\end{abstract}

Key words: advertising models; consumer rights; motivation; attitudes; product quality; advertising.

\section{Resumo}

Por meio de pesquisa quantitativa, descritiva e não exploratória, é determinado o modelo publicitário mais relevante no setor comercial do cantão de Ambato, tomando como referência o banco de dados da ISIC (International Standard Industrial Classification) e o cumprimento das normas direitos do consumidor na aplicação dos modelos que a empresa utiliza no mima, os dez setores comerciais mais importantes do cantão são o objeto de estudo, foram selecionados por 
uma amostra intencional para a proporção de informações mais verdadeiras e principais, a pesquisa é Encontre apoiado por um estudo de campo.

Palavras chave: modelos de publicidade; direitos do consumidor; motivação atitudes; qualidade do produto; publicidade

\section{Introducción}

Mediante la revisión de base de datos del CIIU (Clasificación Industrial Internacional Uniforme) que permitió identificar los sectores comerciales que 457811 existen en los 9 cantones de la provincia de Tungurahua, el estudio está apoyado en una investigación de campo, se ha tomado como referencia de estudio de los diez sectores comerciales más relevantes del cantón. Se determina cual es el modelo publicitario que se utiliza en el sector comercial con respecto a los derechos del consumidor para la satisfacción de sus necesidades ya sea en bienes o servicios. El $32.5 \%$ de empresas del sector comercial del cantón Ambato carecen de un departamento de marketing, publicidad o ventas, lo que genera problemas publicitarios en la comercialización de bienes y servicios. El escaso presupuesto publicitario de las empresas en el sector comercial es el factor que genera una publicidad limitada, de baja calidad y que en ciertas ocasiones no cumple con los derechos del consumidor. Cada empresa del sector comercial debe aplicar un modelo de publicidad específico según el producto o servicio que comercializa garantizando los derechos del consumidor para la satisfacción del cliente.

\section{Modelos Publicitarios}

Según (Sahui, 2008) los modelos publicitarios basados en los factores psicológicos analizan el comportamiento de los consumidores, que forma una reacción estratégica en el marketing para el consumo del mercado. De manera que (Parrado, J.B. Watson y la Publicidad, los inicios de la Psicología del Consumidor, 2013) menciona a J. B. Watson como precursor de los factores psicológicos en la publicidad, que determina técnicas para la creación de publicidad, que cautive la atención del consumidor. Enfatiza en la creatividad de como la publicidad llega al consumidor captando de forma rápida su atención e incitando a la compra inmediata (García M., 2011).

Los modelos publicitarios basados en el aprendizaje o lineales son etapas que el consumidor realiza para llegar a la decisión de compra (García M., 2011). La publicidad basada en el 
aprendizaje se enfoca en medios de comunicación más comunes que existe con la finalidad de crear un recuerdo mayor para el incentivo de compra (Reinares, Reinares, \& Olarte, 2016). La publicidad se encarga de incentivar a la compra al consumidor, con la presentación del producto se convirtiere en información valiosa para la adquisición del producto. El resultado en el aprendizaje de la publicidad es el conocimiento y experiencias que obtiene del producto para influir en el momento de la compra (Rodríguez, Suárez, \& García, 2008).

Según (García N., 2011) menciona a Starch que la publicidad es una opción que las personas pagan por dar a conocer los productos o servicios que ofrecen. De manera que influya al público a dirigirse en actitud y opinión en referencia al mensaje. La característica en el mensaje publicitario es influir a través de él. El modelo Starch mide los anuncios que identifica el consumidor para la construcción de recuerdos que incentiva la conducta de la compra. Al crear recuerdos los consumidores hacen referencia a experiencias emocionales, de esta manera se evalúa al estímulo publicitario según la respuesta emocional que obtuvo el cliente frente a la compra (Guede, 2018).

El modelo AIDA (Atención, Interés, Deseo, Acción) es adaptado a las comunicaciones publicitarias con el fin de atraer la atención al consumidor, a través de la creatividad induciendo finalmente a la compra (Parreño, 2012). El posicionamiento de la empresa y los productos llegan al punto estrella según la matriz BCG, es más fácil al realizar la publicidad, evitando el engaño a los consumidores. Varios modelos pueden ayudar a las empresas a guiar las estrategias publicitarias uno de ellos es el modelo AIDA que ayuda a la captación de atención del consumidor ante el producto o servicio que se intenta transmitir. Este modelo involucra recursos utilizados para los consumidores en el proceso de compra, este involucra valores emocionales, cognitivos y sensoriales que ayuda a distinguir la funcionalidad, seguridad y calidad del producto o servicio que se pretende adquirir.

La atención en el modelo AIDA según (Rodríguez I., 2011) comienza con la parte visual, con el fin de despertar curiosidad ante el producto o servicio expuesto, la publicidad que aplique la empresa ya sea en forma digital o impresa, debe indicar lo más interesante para llamar la atención y despertar el interés del cliente, crear conciencia de la marca a través de comunicación publicitaria, en cuanto sea producto o servicio que esté interesado. 
El interés en referencia a (Barreto, Velásquez, \& Méndez, 2017) surge después de llamar la atención de los consumidores, se intenta mantener una atención de mayor tiempo en el producto o servicio que se pretende vender, es el momento en el cual se crea interés en el cual se explicara los beneficios, técnicas, funcionalidades y características que ofrece, es una atención continua el cual no se encuentra satisfecha una de las formas que más se utiliza es en campañas de marketing online.

El deseo incentiva los sentimientos del consumidor para la posesión del producto o servicio que ofrece la empresa, para persuadir al cliente se necesita convencer y demostrar mediante la exposición del producto que lo necesita para satisfacer sus necesidades, ofreciendo una solución a sus problemas, a continuación, se debe provocar la acción de compra ya sea de un producto o servicio (Jaramillo, Álvarez, \& Guerrero, 2018).

La acción es el último paso en el cual se observa si existe éxito o fracaso de los pasos mencionados anteriormente, el consumidor decide realizar la acción de la compra, el modelo AIDA se ha realizado con éxito al realizar el cierre de la venta, el equipo de ventas de la empresa debe ayudar a que el consumidor se sienta satisfecho con su compra para generar un cliente fiel, evitar cualquier tipo de falla ante el cliente para evitar una compra errada (Barreto, Velásquez, \& Méndez, 2017).

El modelo Dagmar significa “Definig Advertaising Goals for Measured Advertaising Results” el cual se traduce como: definición de objetivos publicitarios para resultados publicitarios medidos, ayuda a fijar objetivos a la empresa con respecto a la publicidad, se dirige a una audiencia ya segmentada en el mercado, con un tiempo determinado, para lograr la medición de resultados de las campañas publicitarias y no resultados de ventas. Afirmando cual fue la eficacia que tuvo dicha campaña publicitaria frente a la empresa. (Rodríguez I., 2011).

En modelo Lavidge-Steiner (Rodríguez I., 2011) menciona que se aplica tres niveles los cuales se menciona a continuación: nivel cognitivo es aquel que el consumidor se informa de los beneficios que se puede obtener mediante la obtención del producto, nivel afectivo es el interés que el consumidor tiene frente al producto e induce al deseo de compra, nivel conativo es la acción que el consumidor tiene con el producto esta puede ser acción de compra o de rechazo. Menciona (López, 2007) que la comunicación a través del marketing se puede utilizar el método de la 
persuasión, la aportación que se obtiene mediante el modelo Lavidge-Steiner son los efectos a largo plazo en la publicidad, cuando el producto es nuevo en el mercado los consumidores deben seguir seis pasos antes de la compra: conciencia, conocimiento, agrado, preferencia convicción y compra.

En referencia a (García M., 2011) los modelos publicitarios basados en motivaciones utilizan factores como; psicología, estímulos y comportamiento para impulsar a los consumidores la compra generando una necesidad. La publicidad se involucra con enseñanzas antiguas, creando estímulos para dominar el comportamiento del consumidor. Al brindar una publicidad con motivación predispone a las personas a actuar mediante sentimientos encontrados, influyendo en el nivel social, económico y de prestigio (Mc Phail, 2012).

En el modelo de Henri Joannis según (Gómez B. , 2017) existe tres etapas que ayudan a la creación de campañas publicitarias como: Elección del eje psicológico, es la observación de las motivaciones que mide la satisfacción que el producto o servicio aporta al consumidor, Creación de la idea central de la campaña, es el concepto que tiene la empresa hacia el producto, informando al consumidor como lo puede beneficiar en dicha compra, el concepto debe ser claro y preciso para no crear confusiones al consumidor con productos similares, Realización del anuncio, publicar el concepto establecido en la etapa anterior según el medio de comunicación que se haya elegido, el mensaje debe ser claro, certero y preciso con el soporte de las campañas publicitarias. En el modelo de Henri Joannis (García M., 2011) también observa que el estudio del comportamiento del consumidor en el ámbito psicológico que se trata de estimular sensaciones con el consumidor, mediante el producto o servicio adquirido satisfacer sus necesidades.

El modelo publicitario basado en las actitudes repercute en el consumidor de manera que modifica las actitudes de los consumidores inclinándose a un solo producto o servicio que sea de su necesidad (García M., 2011). Incrementa el deseo positivo de compra en el consumidor enfocándose en los atributos y ventajas que puede traer dicha adquisición. Los modelos publicitarios varían según las necesidades de los consumidores, mediante estrategias publicitarias los clientes se enfocaran al producto correcto que necesitan (Díaz, González, López, \& Preciado, 2013). 
Según (García M. , 2011) el modelo de Fishbein en la actitud general el individuo deduce los atributos del producto o servicio que se pretende vender mediante sentimientos y creencias, al ser humano lo definen como un animal racional que obtiene información que la procesan con el fin de evaluar, juzgar para llegar a la toma de decisiones frente a lo que disponen a comprar, las creencias y actitudes que el consumidor tiene frente a un producto o servicio es sobre la información que capta al ser transmitida en un medio publicitario. El modelo de Fishbein menciona (Gómez B. , 2017) que tiene una relación entre creencia y actitud que es inculcado en la información de un producto o servicio, este modelo ayuda al cambio de creencias en los consumidores hacia el producto o servicio a través de sus características y/o mejorando su percepción. La modificación de actitudes mediante los atributos que perciben del bien o servicio a adquirir para la facilidad de la compra.

\section{Derechos del consumidor}

La segmentación en referencia a los derechos del consumidor genera protección al ser humano para evitar la estafa de proveedores ya sea en productos o servicios al momento de la compra (Kuri \& Salas, 2015). La segmentación de mercados ayuda a identificar a la población potencial que se considera como meta, existen factores en los que se subdivide a los consumidores como estilo de vida, demográficos (Schiffman \& Lazar, 2010). Existe la necesidad de estudiar las necesidades del consumidor analizadas en la segmentación de mercado, para desarrollar productos y servicios que satisfagan a los mismos.

La investigación de mercado busca las preferencias y necesidades de los consumidores, se la caracteriza por las percepciones del consumidor frente a la publicidad que es desarrollada en el medio (Schiffman \& Lazar, 2010). Es un proceso en el cual se recopila información para la toma de decisiones y solución de problemas, se la realiza de forma sistemática y objetiva. Se investiga información precisa y real sin aplicar ninguna clase de sesgos, la investigación de mercado debe tener información documentada y solidez metodológica (Malhotra, 2016).

La psicología estudia las reacciones de los consumidores en el momento de compra, la compra ayuda a mejorar la autoestima y aumenta el valor de las personas (Vils, Strehlau, Mazzieri, \& Maccari, 2017). El comportamiento del consumidor es analizado por la psicología para estudiar el conjunto de acciones que se obtiene de las personas al momento de decidir algún objeto o 
servicio que sea de su necesidad (Barreto, Velandia, \& Rincón, 2011). Para (Parrado, 2013) según J. B. Watson existen técnicas psicológicas que mejoran la forma de hacer publicidad para llamar la atención del cliente, y tener mayor acogida en el mercado.

(República del Ecuador, 2010) (Congreso Nacional, 2015)

Según (Ley Orgánica de Defensa del Consumidor, 2011) la reparación e indemnización, el cliente tiene derecho cuando el producto adquirido se encuentra dañado o es de mala calidad, ya sea en bienes o servicios, esta acción ayuda a que la víctima pueda exigir al vendedor el dinero o el bien en condiciones óptimas para su uso, la compensación por daños y prejuicios se otorga una indemnización de forma directa a la persona que fue víctima por las partes sufridas. La reparación involucra el restablecimiento y la eliminación de los defectos que se produjo en el producto o servicio adquirido, como seguimiento existe una indemnización como compensación por los daños y molestias que fue causada para el consumidor, el efecto no debe ser solo sustituir el producto o servicio sino debe ser de forma correctiva.

Las acciones administrativas en referencia a la (Ley Orgánica de Defensa del Consumidor, 2011) la empresa tiene el derecho a cumplirlas, la acción administrativa actúa en la representación del Estado generando uso en las facultades regladas, interfiere frente a la misma institución, dispone recursos de reposición, las acciones administrativas son el medio por el cual existe un control de legalidad con cada acto de la administración. En referencia a (Romeral, 2008) es el conjunto de facultades y competencias que son asignadas a las personas que representan a los trabajadores, un derecho a la tutela en los intereses, constituye la garantía efectiva de las facultades conferidas.

Menciona (Lara \& Echaide, 2006) que la publicidad engañosa está prohibida ya que se induce a una publicidad abusiva de un bien o servicio que atenta contra el consumidor, sus derechos y valores en el artículo de la (Ley Orgánica de Defensa del Consumidor, 2011) la publicidad engañosa será sancionada ya que transmite una información de forma fraudulenta ya que induce a un error a los consumidores, afecta el comportamiento económico con el fin de perjudicar a la competencia, no tiene información solida acerca de un producto o servicio que se pretende vender en el mercado, la competencia engañosa tiene como características: precios bajos, mensajes ambiguos, estimulan al consumidor a una compra inmediata, información incompleta. Manipula a 
las personas con el fin de vender sus productos de forma rápida a través de una publicidad que da información falsa muy diferente a la realidad del producto o servicio.

En la (Ley Orgánica de Defensa del Consumidor, 2011) los consumidores tiene derecho a una información adecuada, clara oportuna y completa acerca de los bienes o servicios que las diferentes empresas ofrecen en el mercado, como precios reales, características, condiciones y calidad, con respecto a la información adecuada () menciona que como primera etapa es la información clara y precisa el consumidor, consiste en eliminar la duda, eliminar información que no sea importante, de esa manera se ayudara al consumidor a elegir con mayor facilidad, la información se debe acoplar a los esquemas mentales del consumidor, se debe aprovechar las creencias que las personas tienen esto ayudara a que la transmisión del mensaje sea fuerte al público objetivo.

\section{Metodología}

La investigación realizada determina cual es el modelo publicitario que utilizan en el sector comercial en el cantón de Ambato con respecto a los derechos del consumidor obteniendo datos verídicos mediante los siguientes métodos de investigación utilizando como herramienta para la recolección de datos a la encuesta.

\section{Investigación Cuantitativa}

La investigación cuantitativa según (Barragán, 2003) relaciona directamente a la cantidad y el análisis de los elementos, mediante la recopilación de datos que se obtiene de una población que se ha seleccionado en la investigación a realizar, las herramientas que se utiliza en la investigación cuantitativa son de forma matemáticas y estadísticas para la obtención de resultados. El propósito de la investigación cuantitativa es saber el problema que tan generalizado se encuentra mediante la herramienta que se utilizó para la obtención de información, es una de las investigaciones más utilizadas por compañías ya que ayuda a medir mediante resultados estadísticos y logran interpretar la información para la toma de decisiones (Llopis, 2004).

En referencia a (Schiffman, 2005) se debe trabajar con un modelo numérico para que exista la medición de los resultados y la solución que de desea aplicar, la naturaleza de este modelo es descriptiva que permite que el investigador pueda predecir cuál va a hacer el comportamiento del 
objeto de estudio. En el proyecto a desarrollar la herramienta que se utilizó es la encuesta que nos ayuda a recolectar información verídica para los resultados finales de la investigación.

El sustento del estudio está apoyado en una investigación de campo, mediante la revisión de base de datos del CIIU (Clasificación Industrial Internacional Uniforme) que permitió identificar los sectores comerciales que 457811 existen en los 9 cantones de la provincia de Tungurahua, los cuales tomamos como referencia el cantón Ambato como el sector más comercial.

\section{Investigación Descriptiva}

Se obtiene información mediante un estudio descriptivo que se quiere saber cuándo, dónde, por qué y cómo del objeto de estudio, el objetivo de esta investigación es la descripción de las características de los grupos seleccionados para la investigación en base a un cálculo de la muestra que se obtuvo de la población que se va a investigar, se debe pronosticar los resultados próximos que se van a obtener mediante la investigación (Mohammad, 2005). La investigación descriptiva en referencia a (Bernal, 2006) es un estudio rígido que no se puede manipular como el estudio exploratorio el cual se lo conoce por su flexibilidad, se guía por las preguntas que fueron planteadas por el investigador con referencia a la hipótesis que fue formulada, se dice investigación descriptiva cuando se aprueba la hipótesis mencionada anteriormente.

La formulación de hipótesis existe cuando se detecta el problema en la investigación que se va a realizar, dicho problema debe ser fundamentado mediante el marco teórico, así se determina qué tipo de estudio es el más factible para aplicar en la investigación mediante los datos, población, muestra, etc. Indica la hipótesis que es lo que se trata de probar o buscar mediante explicaciones fundamentadas del fenómeno investigado. Cuando existen investigaciones cuantitativas el método a utilizar puede ser el método deductivo, en el caso de no ser investigación exploratoria se formula la hipótesis estable, siendo el alcance correlacional o explicativo (Gómez M. ,2006).

Se ha identificado la muestra de 373 empresas comerciales que se ha realizado el levantamiento de información para registrar que modelos publicitarios que utilizan con la aplicación de los derechos del consumidor, se ha tomado para el objeto de estudio a los sectores del CIIU más relevantes en el cantón Ambato. 
En esta investigación se ha escogido el muestreo intencional según (Hurtado, 2007) se sugiere tomar ciertas unidades que tienen más realce para obtener información verídica, se destaca la lógica de la significatividad debido a que no se puede tomar de naturaleza equivalente a todas las unidades de estudio, la información que se requiere debe ser abundante. Es por ello que se selecciona las unidades de estudio más representativas en el sector comercial del cantón Ambato.

Tabla 1 Sectores Comerciales

\begin{tabular}{|c|c|c|c|}
\hline CÓDIGO & DESCRIPCIÓN CIIU PRINCIPAL A CUATRO DÍGITOS & $\begin{array}{l}\text { PORCENTAJE DE } \\
\text { PARTICIPACION EN EL } \\
\text { MERCADO }\end{array}$ & $\begin{array}{l}\text { ENCUESTAS } \\
\text { REALIZADAS }\end{array}$ \\
\hline G4520 & Mantenimiento y reparación de vehículos automotores. & $15,1 \%$ & 56 \\
\hline G4530 & $\begin{array}{l}\text { Venta de partes, piezas y accesorios para vehículos } \\
\text { automotores. }\end{array}$ & $4,3 \%$ & 16 \\
\hline G4719 & $\begin{array}{l}\text { Otras actividades de venta al por menor en comercios no } \\
\text { especializados. }\end{array}$ & $3,9 \%$ & 15 \\
\hline G4721 & Venta al por menor de alimentos en comercios especializados. & $7,4 \%$ & 28 \\
\hline G4752 & $\begin{array}{l}\text { Venta al por menor de artículos de ferretería, pinturas y } \\
\text { productos de vidrio en comercios especializados. }\end{array}$ & $5,8 \%$ & 22 \\
\hline G4759 & $\begin{array}{l}\text { Venta al por menor de aparatos eléctricos de uso doméstico, } \\
\text { muebles, equipo de iluminación y otros enseres domésticos en } \\
\text { comercios especializados. }\end{array}$ & $4,6 \%$ & 17 \\
\hline G4761 & $\begin{array}{l}\text { Venta al por menor de libros, periódicos y artículos de papelería } \\
\text { en comercios especializados. }\end{array}$ & $4,1 \%$ & 15 \\
\hline G4771 & $\begin{array}{l}\text { Venta al por menor de prendas de vestir, calzado y artículos de } \\
\text { cuero en comercios especializados. }\end{array}$ & $18,2 \%$ & 68 \\
\hline G4772 & $\begin{array}{l}\text { Venta al por menor de productos farmacéuticos y medicinales, } \\
\text { cosméticos y artículos de tocador en comercios especializados. }\end{array}$ & $5,0 \%$ & 18 \\
\hline G4782 & $\begin{array}{l}\text { Venta al por menor de productos textiles, prendas de vestir y } \\
\text { calzado en puestos de venta y mercados. }\end{array}$ & $11,1 \%$ & 42 \\
\hline \multicolumn{2}{|r|}{ TOTAL } & $99 \%$ & 368 \\
\hline
\end{tabular}

Fuente: CIIU, 2018

*La información que se recolectó mediante encuestas se destacó que son diez los sectores comerciales más destacados en el cantón de Ambato.

\section{Investigación No Experimental}

En referencia a (Toro \& Parra, 2006) las variables no son manipuladas en la investigación no experimental, es decir que las variables independientes no son intencionalmente manipuladas, debido a que se pretende estudiar a las variables independientes en su estado y contexto natural 
para realizar el debido análisis y tomar una decisión acerca de la investigación a realizar, en la investigación no experimental no se construye ninguna situación, al contrario se estudia las situaciones ya existentes, el investigador no tiene opción a manipular las variables. La naturalidad de los fenómenos es observados y analizados en su naturaleza el estudio es sistemático y empírico es decir que las variables independientes no se pueden manipular de ninguna manera (Gómez M., 2006).

Mediante la investigación de los modelos publicitarios aplicados en el sector comercial la variable independiente que se muestra son los modelos publicitarios que se ha identificado cual es la más utilizada, se analiza a través de las reacciones del consumidor en el mercado. La relación con el complimiento de los derechos de consumidor la variable que ha sido parte del estudio en la investigación es los derechos del consumidor debido a que mediante la Ley Orgánica de Defensa del Consumidor se ha dado a conocer la protección que tiene el consumidor en el sector comercial.

Se ha identificado los modelos publicitarios que se aplican en cada uno de los sectores comerciales del cantón Ambato

Tabla 2 Modelos Publicitarios

\begin{tabular}{|l|l|c|}
\hline \multicolumn{2}{|c|}{ MODELOS PUBLICITARIOS } & TOTAL \\
\hline 1 & Modelos Psicológicos & 1 \\
\hline 2 & Modelo de Aprendizaje o Lineal & 4 \\
\hline 3 & Modelo Basado en Motivaciones & 3 \\
\hline 4 & Modelo Basado en Actitudes & 3 \\
\hline
\end{tabular}

Fuente: Elaboración Propia

*En cada modelo publicitario hay un total de los sectores comerciales que utilizan dichos modelos, con 10 sectores comerciales que se ha tomado para la fuente de estudio, cabe recalcar que existe un sector comercial Venta al por menor de productos textiles, prendas de vestir y calzado en puestos de venta y mercados. (G4782) que utiliza dos modelos publicitarios el Modelo de Aprendizaje o Lineal y Modelo Basado en Motivaciones. 
Tabla 3 Porcentaje de Sectores Comerciales y Modelos Publicitarios

\begin{tabular}{|c|c|c|c|c|}
\hline \multirow{2}{*}{$\begin{array}{l}\text { Opción } \\
\text { de M.P. } \\
4\end{array}$} & \multicolumn{3}{|c|}{$\begin{array}{c}\text { Mantenimiento y reparación de vehículos } \\
\text { automotores. (G4520) }\end{array}$} & \multirow{2}{*}{$\begin{array}{c}\text { Modelo Publicitario } \\
\text { Modelo Basado en Actitudes }\end{array}$} \\
\hline & $41,10 \%$ & $35,70 \%$ & $38,40 \%$ & \\
\hline & \multicolumn{3}{|c|}{$\begin{array}{l}\text { Venta de partes, piezas y accesorios para } \\
\text { vehículos automotores. (G4530) }\end{array}$} & \\
\hline \multirow[t]{2}{*}{3} & $87,50 \%$ & $81,30 \%$ & $84,40 \%$ & Modelo Basado en Motivaciones \\
\hline & \multicolumn{3}{|c|}{$\begin{array}{l}\text { Otras actividades de venta al por menor en } \\
\text { comercios no especializados. (G4719) }\end{array}$} & \\
\hline \multirow[t]{2}{*}{4} & $46,70 \%$ & $53,30 \%$ & $50,00 \%$ & Modelo Basado en Actitudes \\
\hline & \multicolumn{3}{|c|}{$\begin{array}{l}\text { Venta al por menor de alimentos en } \\
\text { comercios especializados. (G4721) }\end{array}$} & \\
\hline \multirow[t]{2}{*}{4} & $71,40 \%$ & $75 \%$ & $73,20 \%$ & Modelo Basado en Actitudes \\
\hline & \multicolumn{3}{|c|}{$\begin{array}{l}\text { Venta al por menor de artículos de } \\
\text { ferretería, pinturas y productos de vidrio en } \\
\text { comercios especializados. (G4752) }\end{array}$} & \\
\hline \multirow[t]{2}{*}{1} & $56,20 \%$ & $50,00 \%$ & $53,10 \%$ & Modelos Psicológicos \\
\hline & \multicolumn{3}{|c|}{$\begin{array}{l}\text { Venta al por menor de aparatos eléctricos } \\
\text { de uso doméstico, muebles, equipo de } \\
\text { iluminación y otros enseres domésticos en } \\
\text { comercios especializados. (G4759) }\end{array}$} & \\
\hline \multirow[t]{2}{*}{3} & $76,50 \%$ & $35,30 \%$ & $55,90 \%$ & Modelo Basado en Motivaciones \\
\hline & \multicolumn{3}{|c|}{$\begin{array}{c}\text { Venta al por menor de libros, periódicos y } \\
\text { artículos de papelería en comercios } \\
\text { especializados. (G4761) }\end{array}$} & \\
\hline \multirow[t]{2}{*}{2} & $60 \%$ & $60 \%$ & $60,00 \%$ & Modelo de Aprendizaje o Lineal \\
\hline & \multicolumn{3}{|c|}{$\begin{array}{c}\text { Venta al por menor de prendas de vestir, } \\
\text { calzado y artículos de cuero en comercios } \\
\text { especializados. (G4771) }\end{array}$} & \\
\hline 2 & $61,80 \%$ & $45,60 \%$ & $53,70 \%$ & Modelo de Aprendizaje o Lineal \\
\hline
\end{tabular}




\begin{tabular}{|c|c|c|c|c|}
\hline \multirow[b]{2}{*}{2} & \multicolumn{3}{|c|}{$\begin{array}{c}\text { Venta al por menor de productos } \\
\text { farmacéuticos y medicinales, cosméticos y } \\
\text { artículos de tocador en comercios } \\
\text { especializados. (G4772) }\end{array}$} & \multirow[b]{2}{*}{ Modelo de Aprendizaje o Lineal } \\
\hline & $77,80 \%$ & $38,90 \%$ & $58,4 \%$ & \\
\hline & \multicolumn{3}{|c|}{$\begin{array}{c}\text { Venta al por menor de productos textiles, } \\
\text { prendas de vestir y calzado en puestos de } \\
\text { venta y mercados. (G4782) }\end{array}$} & \\
\hline 2 & $38,20 \%$ & $55,90 \%$ & $47,05 \%$ & Modelo de Aprendizaje o Lineal \\
\hline 3 & $55,90 \%$ & $38,20 \%$ & $47,05 \%$ & Modelo Basado en Motivaciones \\
\hline
\end{tabular}

Fuente: Elaboración Propia

* Se manifiesta que en la Tabla 4 Porcentaje de Sectores Comerciales y Modelos Publicitarios se ha tomado la información de las encuestas realizadas en los diez sectores comerciales más relevantes del cantón de Ambato, identificando cual es el modelo que aplica cada sector.

En la investigación realizada y la recolección de datos indica que el sector comercial G4752 Venta al por menor de artículos de ferretería, pinturas y productos de vidrio en comercios especializados aplica el modelo publicitario basado en factores psicológicos con un porcentaje de 53.1\%. El modelo publicitario de aprendizaje o lineal utilizan los sectores G4761 - Venta al por menor de libros, periódicos y artículos de papelería en comercios especializados con el 60\%,mientra que el sector G4771 - Venta al por menor de prendas de vestir, calzado y artículos de cuero en comercios especializado con el 53.7\%, el 58.4\% pertenece al sector G4772 - Venta al por menor de productos farmacéuticos y medicinales, cosméticos y artículos de tocador en comercios especializados y el 47.1 al sector G4782 - Venta al por menor de productos textiles, prendas de vestir y calzado en puestos de venta y mercados. El modelo publicitario basado en motivaciones utiliza estímulos para impulsar a los consumidores a la compra es aplicado en los sectores G4530 - Venta de partes, piezas y accesorios para vehículos automotores con el 84.4\%, mientras que el 55.9\% pertenece al sector G4759 - Venta al por menor de aparatos eléctricos de uso doméstico, muebles, equipo de iluminación y otros enseres domésticos en comercios especializados, y el sector G4782 - Venta al por menor de productos textiles, prendas de vestir y calzado en puestos de venta y mercados con un porcentaje de $47.1 \%$ cabe recalcar que el sector mencionado utiliza dos modelos publicitarios. El último modelo publicitario basado en actitudes lo utilizan los sectores G4520 - Mantenimiento y reparación de vehículos automotores con el 
38.40\%, el 50\% pertenece al sector G4719 - Otras actividades de venta al por menor en comercios no especializados mientras que el sector G4721 - Venta al por menor de alimentos en comercios especializados tiene el $73.20 \%$

\section{Resultado}

La importancia de un conocimiento publicitario es relevante para las empresas que se encuentran en el mercado, debido a que mediante ese conocimiento se puede crear publicidad que dé a conocer sus productos o servicios que comercializan, en los diez sectores comerciales relevantes del cantón Ambato se indica que el 51.56\% tiene conocimientos acerca de publicidad que aplican es sus empresas, mientras que el $48.44 \%$ no tienen muy claro acerca del tema, por esta razón es que cuando realizan publicidad lo hacen imitando a la competencia. Existen cuatro modelos publicitarios: Modelo basado en factores psicológicos, modelo basado en el aprendizaje o lineales, modelo basado en motivaciones y modelo basado en actitudes de los cuales se ha realizado un estudio de campo para conocer cuál es el modelo que se destaca y puede ser aplicado en los sectores comerciales.

Tabla 5 Porcentaje Modelos Publicitarios

\begin{tabular}{|c|c|c|c|c|}
\hline & $\begin{array}{c}\text { Modelos } \\
\text { Psicológicos }\end{array}$ & $\begin{array}{c}\text { Modelo de } \\
\text { Aprendizaje o } \\
\text { Lineal }\end{array}$ & $\begin{array}{c}\text { Modelo Basado } \\
\text { en Motivaciones }\end{array}$ & $\begin{array}{c}\text { Modelo Basado en } \\
\text { Actitudes }\end{array}$ \\
\hline $\mathbf{1}$ & $53,10 \%$ & $60 \%$ & $84,4 \%$ & $38,40 \%$ \\
\hline $\mathbf{2}$ & $0 \%$ & $53,70 \%$ & $55,9 \%$ & $50 \%$ \\
\hline $\mathbf{3}$ & $0 \%$ & $58,40 \%$ & $47,05 \%$ & $73,20 \%$ \\
\hline $\mathbf{4}$ & $0 \%$ & $47,05 \%$ & $0 \%$ & $0 \%$ \\
\hline & $13,28 \%$ & $54,79 \%$ & $46,84 \%$ & $40,40 \%$ \\
\hline
\end{tabular}

Fuente: Elaboración Propia

El modelo más destacado en el sector comercial del cantón Ambato es el modelo publicitario basado de Aprendizaje o Lineal el cual se sustenta con un porcentaje de 54.79\% que las empresas 
aplican al momento de hacer publicidad, seguido con el modelo publicitario basado en Motivaciones con un porcentaje de $46.84 \%$, el $40.40 \%$ pertenece al modelo publicitario basados en Actitudes y con un menor porcentaje el modelo publicitario Factores Psicológicos de 13.28\% no es muy aplicado por los sectores comerciales que fueron encuestados.

En la investigación se estudia otra variable de derecho del consumidor lo cual se ha realizado el levantamiento de información en los sectores comerciales del cantón Ambato que se encuestó solo el 51.58\% tienen conocimiento de la Ley Orgánica de Defensa del Consumidor el cual menciona cuales son los parámetros que se debe utilizar para realizar una publicidad adecuado, otro tema a tratar es sobre las infracciones que obtendrá el dueño de la empresa si la publicidad es engañosa, si no cumple con las ofertas y condiciones mencionadas en dichas campañas, la indemnización que se debe realizar al consumidor si algún producto o servicio se encuentra defectuoso o si no cumple las expectativas que en la publicidad se planteó. El $48.42 \%$ de los encuestados desconocen el derecho que deben cumplir hacia el consumidor para satisfacer sus necesidades y se convierta en un cliente potencial, también se privan de las acciones que deben seguir para el posicionamiento de la empresa en el mercado. Al tener conocimiento acerca de los modelos publicitarios y la Ley Orgánica de los Derechos del Consumidor se puede realizar una publicidad óptima para la empresa de cada sector comercial en el cantón de Ambato para incrementar las ventas.

\section{Discusión}

Mediante el análisis de campo se ha hecho una selección de los sectores más representativos del cantón Ambato para realizar la indagación de información, que ayudaron a identificar cual es el modelo publicitario más relevante que es utilizado en cada uno de los sectores comerciales, el modelo publicitario de Aprendizaje o Lineal es el que destaca en esta investigación y es óptimo para que sea uno de los principales para el uso de las empresas del sector comercial. Mediante la Ley Orgánica de los Derechos del Consumidor se ha relacionado que los modelos publicitarios pueden ser ayuda tanto para empresa que comercializa productos o servicio como para el consumidor, generando la protección e integridad de las personas. el conocimiento sobre la publicidad ayuda a las personas a realizar campañas adecuadas para atraer clientes y captarlos de una mejor forma para que realice una compra rápida y precisa. 


\section{Conclusiones}

Se identificó el que modelo publicitario que utiliza cada empresa del sector comercial y que se garantizó que se aplique los derechos del consumidor y este sea satisfactorio en manera que realice su compra siendo como influencia la publicidad que la empresa ha aplicado en el marcado, generando si un elevado número de ventas

Luego de la revisión se concluye que los modelos publicitarios más destacado, es el modelo de Aprendizaje o Lineal que se enfoca en el medio por el cual se realiza la publicidad y los resultados que este provoca en la empresa.

Existe conocimiento acerca de la Ley Orgánica de los Derechos del Consumidor y la protección que tiene el consumidor al momento de realizar alguna compra en un establecimiento, y sanciones que puede tener la empresa si el producto o servicio no tiene las características que fue trasmitida en la publicidad.

\section{Referencias Bibliográficas}

Barragán, R. (2003). Guía para la formulación y ejecución de proyectos de investigación. La Paz: PIEB.

Barreto, I., Velandia, A., \& Rincón, J. (2011). Estrategias metodológicas para el análisis de datos textuales: aplicaciones en psicología del consumir. Suma Psicológica, 9.

Barreto, J., Velásquez, A., \& Méndez, M. (2017). Organización de eventos como herramienta de comunicación de marketing para la radio en la ciudad de Bogotá. UNIEMPRESARIAL, 28.

Bernal, C. (2006). Metodología de la Investigación. México: Pearson Educación.

Congreso Nacional. (2015). Ley Orgánica del Defensa del Consumidor. Lexis.

Díaz, C., González, R., López, E., \& Preciado, C. (2013). Mercadotecnia. Guadalajara: Editorial universitaria.

García, M. (2011). Las claves de la Publicidad. Madrid: Esic Editorial.

García, N. (2011). Educación en publicidad, un acercamiento al contexto Colombiano. Designia, 12. 
Gómez, B. (2017). Fundamentos de la Publicidad. Madrid: ESIC.

Gómez, M. (2006). Inducción a la metodología de la investigación científica. Argentina: Editorial Brujas.

Guede, A. (2018). Grado en Publicidad y Relaciones Públicas Curso 2017 - 2018. UVa SEGOVIA, 52.

Hurtado, J. (2007). El proyecto de investigación. Caracas: Ediciones Quirón.

Jaramillo, L., Álvarez, J., \& Guerrero, L. (2018). Aplicación del neuromarketing por medio del modelo de presentación AIDA en la gestión de ventas del organismo evaluador "Juan Bautista Aguirre". Observatorio de la Economía Latinoamericana, 16.

Kuri, D., \& Salas, E. (2015). Los derechos del consumidor en el Ecuador. Nómadas, 10.

Lara, R., \& Echaide, J. (2006). Consumo y Derecho. Madrid: ESIC.

Ley Orgánica de Defensa del Consumidor. (13 de octubre de 2011). Ley Orgánica de Defensa del Consumidor. Obtenido de Ley Orgánica de Defensa del Consumidor: https://www.industrias.gob.ec/wp-content/uploads/2015/04/A2-LEY-ORGANICA-DEDEFENSA-DEL-CONSUMIDOR.pdf

Llopis, R. (2004). Grupos de Discusión. Madrid: ESIC.

López, B. (2007). Publicidad emocional Estrategias Creativas. Madrid: ESIC.

Malhotra, N. (2016). Investigación de mercados: Conceptos esenciales. México: Pearson Educación.

Mc Phail, E. (2012). PUBLICIDAD, HÁBITOS Y MOTIVACIONES. Razón y Palabra, 24.

Mohammad, N. (2005). Metodología de la investigación. México: Limusa Noriega Editores.

Parrado, F. (2013). J.B. Watson y la Publicidad, los inicios de la Psicología del Consumidor. Revista Colombia de Psicología, 6.

Parrado, F. (2013). J. B. Watson y la Publicidad, los inicios de la Psicología del Consumidor. Revista Colombiana de Psicología, 7. 
Parreño, J. (2012). Determinantes de la eficacia publicitaria actual: el Modelo AMBER.

Questiones Publicitarias, 17.

Reinares, E., Reinares, P., \& Olarte, C. (2016). Formatos de publicidad no convencional en televisión versus spots: un análisis basado en el recuerdo. Historia y Comunicación Social, 22.

República del Ecuador. (2010). Instituto Nacional de Estadística y Censos. Anexo N7:

Clasificación de Actividades Económicas CIIU-4.0.

Rodríguez, I. (2011). Estrategias y Técnicas de Comunicación. Barcelona: UOC.

Rodríguez, I., Suárez, A., \& García, M. (2008). Dirección publicitaria. Editorial UOC.

Romeral, J. (2008). El Delegado de Prevención. España: Lex Nova.

Sahui, J. (2008). Influencia de los factores psicológicos en la conducta del consumidor. Tecsistecatl, 15.

Schiffman. (2005). Comportamiento del Consumidor. Madrid: Pearson Prentice Hall.

Schiffman, L., \& Lazar, L. (2010). Comportamiento del consumidor. México: Pearson Educación.

Toro, I., \& Parra, R. (2006). Método y conocimiento Metodología de la Investigación. Medellín: Fondo Editorial.

Vils, L., Strehlau, S., Mazzieri, M., \& Maccari, E. (2017). PERSPECTIVAS DA PSICOLOGIA EVOLUCIONISTA EM COMPORTAMENTO DO CONSUMIDOR. Revista Brasileira de Marketing, 14. 